\title{
Incapacidade gerada pela dor osteomuscular em aluno de Odontologia
}

\author{
Incapacity generated by musculoskeletal pain in dental students \\ Incapacidad generada por el dolor osteomuscular en estudiantes de Odontología \\ Renata Reis dos SANTOS \\ Cléa Adas Saliba GARBIN \\ Tânia Adas SALIBA \\ Renata Colturato Joaquim GATTO \\ Artênio José Isper GARBIN \\ Programa de Pós-Graduação em Odontologia Preventiva e Social, Faculdade de Odontologia de Araçatuba \\ Univ. Estadual Paulista - UNESP, 16015-050 Araçatuba-SP, Brasil
}

\begin{abstract}
Resumo
Introdução: A sintomatologia dolorosa no sistema músculo-esquelético é uma grande preocupação entre os cirurgiões-dentistas. Objetivo: Avaliar a incapacidade gerada pela dor e o nível de intensidade da dor, verificando se existe uma correlação entre essas. Metodologia: Foi realizado um estudo transversal com alunos de Odontologia que cursaram ao menos uma disciplina clínica (n=303). Os estudantes foram investigados sobre a presença de dor, utilizando o Questionário Nórdico. Aos alunos sintomáticos foi aplicado também o Pain Disability Questionnaire (PDQ) e a Escala Numérica da Dor. A análise dos resultados foi realizada por meio do teste de correlação de Spearman. Resultados: Consentiu participar da pesquisa, um total de 241 alunos e destes, 199 (82,6\%) apresentaram sintomatologia da dor. A incapacidade gerada pela dor foi classificada como moderada em 76,9\% dos alunos. Em relação a escala de dor, a média encontrada nos alunos foi de 2,19. A intensidade da dor foi classificada com moderada em 11,5\%, mínima em 55,8\% dos alunos, e nula em 32,7\%. Foi encontrada uma correlação positiva e estatisticamente significante entre a maioria das questões do PDQ e a Escala de dor. A correlação entre os domínios do PDQ (CF, CP e Total) e a Escala de dor se mostrou moderada, positiva e estatisticamente significante ( $\rho=0.433$ e p $\leq 0,01$; $\rho=0.307$ e $p \leq 0,01 ; \rho=0,456$ e $p \leq 0,01)$. Conclusão: Conclui-se que existe uma correlação positiva entre a incapacidade gerada pela dor e a intensidade da dor, e estas são classificadas como: moderada e mínima, respectivamente, na maioria dos alunos.

Descritores: Dor Musculoesquelética; Estudantes de Odontologia; Odontologia do Trabalho; Saúde do Trabalhador; Riscos Ocupacionais.

\section{Abstract}

Introdução: Painful symptomatology of the musculoskeletal system is a great preoccupation among dental surgeons. Objective: To assess the incapacity generated by the pain and its level of intensity, and verify whether there is a correlation between them. Methods: A cross-sectional study was performed with dental students who had taken at least one clinical course $(n=303)$. The Nordic Questionnaire was used to investigate the presence of pain in the students. In symptomatic students, the Pain Disability Questionnaire (PDQ) and the Numeric Pain Scale were also applied. The analysis of the results was performed by using Spearman's correlation test. Results: A total of 241 students consented to participate in the research, of whom $199(82.6 \%)$ presented pain symptomatolgy. Incapacity generated by pain was classified as moderate in $76.9 \%$ of the students. As for the Pain Scale, the mean score found in the students was of 2.19. Pain intensity was classified as moderate in $11.5 \%$ and mild in $55.8 \%$ of the students, while $32.7 \%$ claimed no pain. We found a positive and statistically significant correlation between most PDQ questions and the Pain Scale. The correlation between PDQ domains (CF, PC and Total) and the Pain Scale were moderate, positive and statistically significant $(\rho=0.433$ and $p \leq 0.01 ; \rho=0.307$ and $p \leq 0.01 ; \rho=0.456$ and $p \leq 0.01)$. Conclusion: We conclude there is a positive correlation between the incapacity generated by pain and pain intensity, and these are classified as moderate and mild, respectively, in most students.
\end{abstract}

Descriptors: Musculoskeletal Pain; Students, Dental; Occupational Dentistry; Occupational Health; Occupational Risks.

\section{Resumen}

Introducción: La sintomatología dolorosa en el sistema musculoesquelético es una gran preocupación entre los cirujanos-dentistas. Objetivo: Evaluar la incapacidad generada por el dolor y el nivel de intensidad del dolor, verificando si existe una correlación entre esas. Metodología: Se realizó un estudio transversal con alumnos de Odontología que cursaron al menos una disciplina clínica $(\mathrm{n}=303)$. Los estudiantes fueron investigados sobre la presencia de dolor, utilizando el Cuestionario Nórdico. A los alumnos sintomáticos se aplicó también el Pain Disability Questionnaire (PDQ) y la Escala Numérica del Dolor. El análisis de los resultados fue realizado por medio de la prueba de correlación de Spearman. Resultados: Consentió participar de la investigación, un total de 241 alumnos y de éstos, 199 (82,6\%) presentaron sintomatología del dolor. La incapacidad generada por el dolor fue clasificada como moderada en el 76,9\% de los alumnos. En relación a la escala de dolor, la media encontrada en los alumnos fue de 2,19. La intensidad del dolor fue clasificada con moderada en el 11,5\%, mínima en el 55,8\% de los alumnos, y nula en el 32,7\%. Se encontró una correlación positiva y estadísticamente significativa entre la mayoría de las cuestiones del PDQ y la Escala de dolor. La correlación entre los dominios del PDQ (CF, CP y Total) y la Escala de dolor se mostró moderada, positiva y estadísticamente significativa $(\rho=0.433 \mathrm{ep} \leq 0,01 ; \rho=0.307 \mathrm{ep} \leq 0,01 ; \rho=0,456 \mathrm{ep} \leq 0,01)$. Conclusión: Se concluye que existe una correlación positiva entre la incapacidad generada por el dolor y la intensidad del dolor, y éstas se clasifican como: moderada y mínima, respectivamente, en la mayoría de los estudiantes.

Descriptores: Dolor Musculoesquelético; Estudiantes de Odontología; Odontología del Trabajo; Salud Laboral; Riesgos Laborales.

\section{INTRODUÇÃO}

As desordens osteomusculares são consideradas as maiores causas de morbidade e incapacidade em adultos, e representam a principal causa de invalidez em diferentes países ${ }^{1,2}$. Dentre elas, destaca-se a LER (Lesões por Esforço Repetitivo), atualmente renomeadas de DORT (Distúrbios Osteomusculares Relacionados ao Trabalho). Estes distúrbios afetam, principalmente, a região do pescoço, da cintura escapular e dos membros superiores. Sua origem é proveniente da atividade laboral e ocupam o segundo lugar das causas de doenças entre os trabalhadores brasileiros $^{3,4}$.

Assim, essa temática tem alertado a sociedade brasileira, pois houve um expressivo aumento no número de trabalhadores afastados por desordens osteomusculares, que passou a ser considerado um problema de saúde pública ${ }^{5}$. Grande parte das aposentadorias por invalidez é decorrente dessas patologias. Uma parcela significativa de 
pacientes diagnosticados como portadores de distúrbios osteomusculares, desenvolve dor crônica, levando à limitação ou incapacidade para a reinserção produtiva, constituindo um grande desafio para pacientes e médicos, e um alto custo social e econômico para o país ${ }^{6}$. Além disso, as repercussões da dor crônica ultrapassam os limites do ambiente de trabalho, pois interferem na rotina dos indivíduos acometidos, devido à dificuldade para dormir, as alterações de humor e a incapacidade para a realização de tarefas simples do seu cotidiano, podendo até mesmo interferir nas atividades de autocuidado, o que gera um intenso sofrimento psicossocial $^{7}$.

Essas desordens são associadas a múltiplos fatores de risco, são eles: físicos, psicossociais, organização do trabalho e sociocultural. Os tipos e os níveis de riscos variam de acordo com as características individuais e as exigências profissionais ${ }^{8}$.

Alguns grupos de trabalhadores podem se tornar mais propensos ao aparecimento de dores musculoesqueléticas, devido aos riscos ocupacionais ${ }^{9}$. A prática odontológica é uma profissão de alto risco e todos os anos, um grande número de dentistas é acometido por distúrbios musculoesqueléticos relacionados ao trabalho ${ }^{10}$. Essas lesões podem ser ocasionadas pela dificuldade em estabelecer um equilíbrio postural para desempenhar suas funções durante longas horas de trabalho e em posições estáticas, além disso, a falta de intervalos e os movimentos recorrentes e repetitivos também agravam a situação ${ }^{9,11}$.

Cerca de $75 \%$ dos profissionais de Odontologia estão expostos ao risco de desenvolvimento de distúrbios osteomusculares relacionados ao trabalho $^{12}$. A sintomatologia dolorosa, comumente relatada pelo cirurgião-dentista, pode se desenvolver ainda no período de graduação e seguir durante sua vida profissional, caso medidas preventivas ou corretivas não sejam implementadas ${ }^{13}$.

Estudantes de Odontologia trabalham no mesmo ambiente e reproduzem os mesmos comportamentos de trabalho dos cirurgiões-dentistas. Porém as habilidades para enfrentar problemas ou dificuldades no trabalho são limitadas no aluno. Além disso, existe a presença de estressores psicossociais provenientes do ambiente escolar (sobrecarga de informação, ansiedade, desempenho escolar e acessibilidade dos professores e funcionários) somada a carga horária excessiva, atualmente preconizada no curso de graduação em Odontologia ${ }^{12,14}$. O efeito cumulativo resultante pode predispor os alunos às desordens osteomusculares ${ }^{12}$.

Diante do risco, aos quais os alunos de Odontologia estão expostos, é necessário verificar se a presença da sintomatologia dolorosa está gerando incapacidade para o trabalho e, também em suas atividades diárias.
Assim, este trabalho tem por objetivo, verificar a correlação entre a intensidade da dor e a incapacidade que a mesma causa nos alunos de Odontologia que tem sintomatologia de dor positiva.

\section{MATERIAL E MÉTODO}

Foi realizado estudo transversal com alunos do curso de Odontologia, regularmente matriculados em uma Universidade Estadual, localizada no interior do Estado de São Paulo, os quais deveriam ter cursado ao menos uma disciplina que contemplasse em seu plano de ensino a prática de atividades clínicas. A coleta foi realizada num período de três meses e haviam 303 alunos matriculados que atendiam a este critério.

Foram excluídos os portadores de alguma deficiência física congênita ou adquirida, que envolva membros superiores ou inferiores, articulares ou de coluna; gestantes; lactantes e pessoas que não consentiram em participar da pesquisa.

Todos os participantes assinaram o Termo Consentimento Livre e Esclarecido, para que qualquer dúvida fosse esclarecida sobre a participação voluntária na pesquisa. O estudo foi aprovado pelo comitê de ética em pesquisa da faculdade estudada, atendendo todas as normativas nacionais e internacionais que envolvem seres humanos.

Os participantes responderam primeiramente ao Questionário Nórdico de Sintomas Osteomusculares. Aqueles que apresentaram sintomatologia dolorosa nos últimos 12 meses, responderam ao Pain Disability Questionnaire e a escala numérica de dor, para verificar o quanto a dor atrapalhava em suas atividades diárias.

O Questionário Nórdico de Sintomas Osteomusculares é um instrumento validado, e apresenta uma confiabilidade que varia de 0,88 a 1 , segundo o coeficiente de Kappa. Neste estudo foi utilizada sua versão adaptada para o Português ${ }^{15}$. Este instrumento é utilizado internacionalmente e aceito para avaliação das desordens osteomusculares, sendo composto por questões sobre nove áreas do corpo (três áreas das extremidades superiores, três áreas das extremidades inferiores e três áreas da coluna). As regiões avaliadas são: pescoço, ombro, cotovelo, antebraço, punho/mão/dedo, região dorsal, região lombar, quadril/coxa, joelho e tornozelo/pé. O participante relata se já teve alguma experiência de dor ou desconforto em alguma dessas regiões durante os 12 últimos meses.

\section{$\mathrm{O}$ instrumento The Pain Disability} Questionnaire $(P Q D)$ tem como finalidade medir a incapacidade gerada pela dor. A versão brasileira do instrumento foi adaptada culturalmente por Giordano et $\mathrm{al}^{16}$, e sua confiabilidade foi estimada por meio da consistência interna e da estabilidade (teste-reteste) com alfa de Cronbach de 0,86. 
O PDQ é composto por 15 questões que são divididas em dois componentes: funcional $\mathrm{e}$ psicossocial. O componente funcional é composto por 9 questões $(1,2,3,4,5,6,7,12$, e 13) que refletem o estado funcional e a incapacidade, incluindo questões sobre a intensidade da dor, o autocuidado, o levantar, o caminhar, o manter-se em pé, o dormir, a participação na vida social, e o viajar. O componente psicossocial é composto por 6 questões $(8,9,10,11,14$, e 15$)$ relacionados à variáveis sociais tais como, mudança financeira, dependência de medicamentos, visita ao hospital, relações pessoais, mudança de humor e problemas psicológicos ${ }^{17}$.

Cada item é pontuado de 0 a 10 , usando uma escala visual analógica de 10 centímetros, onde os valores mais altos representam maior deficiência. $\mathrm{O}$ componente funcional tem uma pontuação máxima de 90 pontos, e o componente psicossocial uma pontuação máxima de 60 pontos. A pontuação total do PDQ varia de 0 a 150 , e classifica o grau de deficiência da seguinte forma: 0 , sem deficiência; 1 70, deficiência leve; 71-100, deficiência moderada; 101-130, deficiência grave; e 131- 150, extrema deficiência.

Também foi utilizada uma escala numérica com pontuação de 0 a 10 , para avaliar a intensidade da dor. O zero significa ausência de dor e o dez significa pior dor possível. A escala de dor foi dividida em quatro categorias: Nula (0-1); Mínima (2-3); Moderada (4-7); Intensa (8-10).

Todos os questionários foram entregues ao final das aulas teóricas, afim de não atrapalhar as atividades de ensino. Os questionários foram distribuídos aos participantes por alunos de pósgraduação, previamente treinados, que explicaram e esclareceram dúvidas, evitando erros de preenchimento e de compreensão.

Os resultados foram tabulados em uma planilha eletrônica e os testes foram realizados com o auxílio do programa SPSS - "Statistical Package for the Social Sciences" - versão 21.0, por um especialista da área. Como os dados não tiveram uma distribuição conforme a curva normal (rejeição da hipótese de normalidade dos dados), foi utilizado o teste não paramétrico de coeficiente de correlação de Spearman, para avaliar a correlação entre a intensidade da dor e a incapacidade, em alunos sintomáticos. Foi empregado o coeficiente alfa de Cronbach para avaliar a consistência interna do instrumento que permitiu o cálculo de escores de domínios. Considerou-se um valor satisfatório quando $>0,70$.

\section{RESULTADOS}

Dos 303 alunos que estavam matriculados no curso de Odontologia, e cursaram ao menos uma disciplina clínica, 241 participaram da pesquisa. A perda foi devido à ausência do aluno no dia da coleta de dados, ao não consentimento em participar da pesquisa, e àqueles que atendiam aos critérios de exclusão (portadores de alguma deficiência física congênita ou adquirida, que envolvia membros superiores ou inferiores, articulares ou de coluna; gestantes e lactantes).

A média de idade foi de 22 anos e $62,7 \%$ eram mulheres. A presença de dor nos últimos 12 meses foi observada em 199 dos participantes $(83,0 \%)$. Mais da metade dos entrevistados $(51,5 \%)$ indicaram presença de dores no pescoço, 49,4\% relataram dor na parte superior das costas, $38,4 \%$ no pulso/mão e 49,8\% região lombar. Dores musculoesqueléticas nas extremidades inferiores (quadris/coxas, joelhos, panturrilha/perna, tornozelos/pés), foram relatadas por menos de $30 \%$ dos entrevistados.

O questionário PDQ apresentou índices de consistência interna confiáveis, sendo encontrado um valor de alfa de Cronbach de 0.788 para o Componente Funcional, de 0.708 para o Componente Psicossocial e de 0.838 para o PDQ total.

A maioria dos alunos $(76,88 \%)$ que passou por experiência de dor nos últimos 12 meses $(n=199)$, teve a incapacidade, por ela gerada, classificada como moderada, e o restante foi classificado como sem incapacidade. $O$ grau mais elevado de incapacidade não foi encontrado nesta amostra.

Em relação a escala de dor, a média encontrada foi de $2,19 \%$. A intensidade de dor foi classificada como moderada em $11,5 \%$, como mínima em $55,8 \%$, e nula em $32,7 \%$ dos alunos (Tabela 1).

Tabela 1. Distribuição das frequências das categorias do PDQ e da Escala de dor nos alunos de Odontologia. Araçatuba, 2013

\begin{tabular}{lcc}
\hline \multicolumn{1}{l}{ n } & \% \\
\hline PDQ & & \\
\hline Sem incapacidade & 46 & 23.12 \\
Moderada & 153 & 76.88 \\
\hline Escala de dor & & \\
\hline Nula & 63 & 32.7 \\
Mínima & 111 & 55.8 \\
Moderada & 23 & 11.5 \\
\hline TOTAL & $\mathbf{1 9 9}$ & $\mathbf{1 0 0 , 0}$ \\
\hline
\end{tabular}

Quando analisadas as médias de cada questão do PDQ, todas estavam abaixo de 0,6 . Foi encontrada correlação positiva e estatisticamente significante entre a maioria das questões do PDQ e a Escala de Dor. As questões que não apresentaram correlação foram duas do componente psicossocial (3 e 13) e duas do componente funcional (8 e 11) (Tabela 2).

A média total do PDQ foi de 4,6, para o domínio componente funcional $(\mathrm{CF})$ foi de 3,5 e para o domínio componente psicossocial (CP) foi de 1,2. A correlação entre os domínios do PDQ (CF, CP e Total) e Escala de Dor se mostraram moderadas, positivas e estatisticamente significantes, ou seja, à 
medida que a Escala de Dor aumenta, o PDQ também aumenta. Destaca-se um maior coeficiente de correlação da Escala de Dor com o PDQ Total (Tabela 3).

Tabela 2. Correlação entre as questões do PDQ e Escala de Dor nos alunos de Odontologia

\begin{tabular}{|c|c|c|c|c|c|c|}
\hline \multicolumn{5}{|c|}{ PDQ } & \multicolumn{2}{|c|}{ Escala de Dor } \\
\hline Question & Média & DP & $\begin{array}{l}\text { Menor } \\
\text { Valor }\end{array}$ & $\begin{array}{c}\text { Maior } \\
\text { valor }\end{array}$ & Correlação & $p$ \\
\hline $\begin{array}{l}\text { 1.A dor que você sente interfere no seu trabalho } \\
\text { dentro e fora de casa? }\end{array}$ & 0.76 & 0.90 & 0 & 4 & $0.365^{*}$ & $<0.001$ \\
\hline $\begin{array}{l}\text { 2.A dor que você sente interfere na realização } \\
\text { de seus cuidados pessoais (como tomar banho, } \\
\text { vestir-se, etc.)? }\end{array}$ & 0.15 & 0.50 & 0 & 4 & $0.157^{*}$ & 0.029 \\
\hline $\begin{array}{l}\text { 3.A dor que você sente interfere na sua } \\
\text { locomoção? }\end{array}$ & 0.21 & 0.53 & 0 & 3 & 0.066 & 0.360 \\
\hline $\begin{array}{l}\text { 4.A dor que você sente afeta sua capacidade de } \\
\text { sentar-se ou ficar em pé? }\end{array}$ & 0.40 & 0.77 & 0 & 4 & $0.265^{*}$ & $<0.001$ \\
\hline $\begin{array}{l}\text { 5.A dor que você sente afeta sua capacidade de } \\
\text { levantar objetos acima de sua cabeça, segurar } \\
\text { objetos ou alcançar coisas? }\end{array}$ & 0.32 & 071 & 0 & 5 & $0.215^{*}$ & 0.003 \\
\hline $\begin{array}{l}\text { 6.A dor que você sente afeta sua capacidade de } \\
\text { levantar objetos do chão, curvar-se inclinar-se } \\
\text { ou agachar-se? }\end{array}$ & 0.57 & 080 & 0 & 4 & $0.359 *$ & $<0.001$ \\
\hline $\begin{array}{l}\text { 7. A dor que você sente afeta sua capacidade de } \\
\text { caminhar ou correr? }\end{array}$ & 0.56 & 0.90 & 0 & 4 & $0.189 *$ & 0.008 \\
\hline $\begin{array}{l}\text { 8. A sua renda mensal diminuiu desde que a sua } \\
\text { dor começou? }\end{array}$ & 0.03 & 0.22 & 0 & 2 & 0.024 & 0.740 \\
\hline $\begin{array}{l}\text { 9. Você tem que tomar medicamentos todos os } \\
\text { dias para controlar a sua dor? }\end{array}$ & 0.21 & 0.63 & 0 & 4 & $0.208 *$ & 0.004 \\
\hline $\begin{array}{l}\text { 10. A dor que você sente o (a) obriga a procurar } \\
\text { médicos com muito mais frequiência do que } \\
\text { antes da sua dor começar? }\end{array}$ & 0.27 & 0.62 & 0 & 4 & $0.254 *$ & $<0.001$ \\
\hline $\begin{array}{l}\text { 11. A dor que você sente interfere na sua } \\
\text { capacidade de ver as pessoas que são } \\
\text { importantes para você tanto quanto gostaria? }\end{array}$ & 0.13 & 0.39 & 0 & 2 & 0.109 & 0.129 \\
\hline $\begin{array}{l}\text { 12. A dor que você sente interfere nas atividades } \\
\text { de recreação e lazer que são importantes para } \\
\text { você? }\end{array}$ & 0.43 & 0.79 & 0 & 4 & $0.243 *$ & 0.001 \\
\hline $\begin{array}{l}\text { 13. Você precisa de ajuda dos seus familiares e } \\
\text { amigos para terminar suas tarefas diárias } \\
\text { (incluindo tanto trabalho fora de casa quanto } \\
\text { doméstico) por causa da sua dor? }\end{array}$ & 0.07 & 0.34 & 0 & 3 & 0.034 & 0.633 \\
\hline $\begin{array}{l}\text { 14. Atualmente você se sente mais deprimido, } \\
\text { tenso ou ansioso do que antes da sua dor } \\
\text { começar? }\end{array}$ & 0.35 & 0.72 & 0 & 3 & $0.228 *$ & 0.001 \\
\hline $\begin{array}{l}\text { 15. Você apresenta problemas emocionais } \\
\text { causados pela dor, que interferem no } \\
\text { relacionamento familiar, na vida social ou nas } \\
\text { atividades do trabalho? }\end{array}$ & 0.17 & 0.48 & 0 & 3 & $0.286^{*}$ & $<0.001$ \\
\hline
\end{tabular}

Tabela 3. Correlação entre as questões os componentes PDQ, PDQ total e Escala de Dor nos alunos de Odontologia. Araçatuba, 2013

\begin{tabular}{ccccc}
\hline \multirow{2}{*}{ PDQ } & \multicolumn{5}{c}{ Escala de dor } \\
\cline { 2 - 5 } & Média & DP & Correlação & $\boldsymbol{p}$ \\
\hline $\begin{array}{c}\text { Condição } \\
\text { funcional }\end{array}$ & 3.45 & 3.93 & $0.433^{*}$ & $<0.001$ \\
$\begin{array}{c}\text { Componente } \\
\text { Psicossocial }\end{array}$ & 1.15 & 2.04 & $0.307^{*}$ & $<0.001$ \\
PDQ Total & 4.60 & 5.39 & $0.456^{*}$ & $<0.001$ \\
\hline
\end{tabular}

\section{DISCUSSÃO}

Os achados desta pesquisa revelaram um alto índice de estudantes com sintomatologia dolorosa, mais de $80 \%$ relataram presença de dor nos últimos 12 meses. Esse fato torna-se relevante, tendo em vista que a amostra foi composta por alunos do curso noturno e integral, e por estudantes que ainda não cursaram todas as disciplinas clínicas, ou seja, as horas de atendimento eram reduzidas. Outro dado importante revelado pelo estudo refere-se ao fato de que a maior parte dos alunos com sintomatologia dolorosa apresentou incapacidade classificada como moderada, demonstrando assim, a interferência da 
dor na prática clínica, antes mesmo de estarem inseridos no mercado de trabalho.

Assim, algumas medidas preventivas devem ser adotadas pelos alunos, para evitar a frequência dos sintomas dolorosos e posteriormente o diagnóstico de DORT. Uma alternativa é a ginástica laboral, incluindo os alongamentos, massagens, relaxamento físico e psicológico e, se necessário, fisioterapia ${ }^{18}$.

Em relação ao questionário utilizado, tem-se que, o PDQ é um instrumento relativamente novo, criado em 2002, e que não se limita a avaliar apenas o estado funcional, pois, por meio dele, é possível avaliar também as variáveis psicossociais, que desempenham um papel fundamental no desenvolvimento e na manutenção da dor crônica e da incapacidade relacionada à ela ${ }^{19}$.

Dessa forma, com exceção das questões 3 e 13, do componente psicossocial, e das questões 8 e 11 do componente funcional, houve correlação entre todas as questões do PDQ e da escala numérica da dor. A correlação de dor aos componentes psicossociais, que se referem à locomoção e à necessidade de ajuda para execução de tarefas, talvez não tenha sido encontrada, devido aos níveis de incapacidade serem moderados neste estudo, não interferindo assim, em tarefas rotineiras e na movimentação destes alunos. Quando observado o componente funcional, relativo às questões financeiras, o fato de não haver interferência das dores em seu processo de geração de renda pode ser explicado, devido à maioria dos alunos não trabalharem e terem seus estudos financiados por pais ou familiares.

A correlação entre os escores do PDQ Total e a Escala Numérica da Dor e do PDQ e suas dimensões, mostraram-se positivas, moderadas e estatisticamente significantes, com valores próximos ao encontrado nos estudos de validação original do instrumento $(\mathrm{r}=0,44)^{20}$ e também, nos estudos da validação para o português $(\mathrm{r}=0,36)^{16}$, porém uma correlação menor ao encontrado em pacientes com dor lombar ${ }^{21}$.

Ao se estabelecer a relação entre intensidade da dor e incapacidade, outras variáveis devem ser consideradas, tais como frequência e localização da dor, presença de depressão, crenças sobre a dor, uso de medicamentos, entre outros ${ }^{16,22,23}$. A incapacidade gerada pela dor envolve a interação entre aspectos físicos, psicológicos e sociais. ${ }^{16}$ Entender como a escala de dor está associada à incapacidade, tem importantes implicações para o tratamento, e também, na construção de programas de prevenção das desordens e promoção da saúde.

Os profissionais da área de Odontologia são mais vulneráveis ao desenvolvimento de patologias osteomusculares, e por isso, devem estar atentos aos primeiros sinais de alteração, para que possam prevenir e tratá-las o mais cedo possível, evitando o comprometimento de estruturas anatômicas importantes para a profissão, aumentando a longevidade laboral ${ }^{3}$.

Diante do exposto, deve-se ressaltar a importância deste estudo, pois os hábitos posturais incorretos podem ser desenvolvidos durante a graduação e intensificados ao longo da vida profissional, portanto a correção da postura e inserção de hábitos saudáveis, como a prática de exercícios físicos e medidas preventivas, como a ginastica laboral devem ser estimuladas na fase de formação do aluno, evitando assim, problemas futuros como o desenvolvimento de dor crônica, o abandono prematuro da profissão, a aposentadoria por invalidez, o absenteísmo, entre outros problemas.

\section{CONCLUSÃO}

Os estudantes participantes desta pesquisa apresentaram um alto índice de sintomatologia dolorosa. Houve uma correlação positiva entre a intensidade de dor e a incapacidade gerada por ela. Este achado é de grande valia, tendo em vista o comprometimento desses futuros profissionais. A graduação deve capacitar os estudantes para terem hábitos saudáveis que permitam a longevidade profissional.

Embora os achados deste estudo não possam ser generalizados, é importante conhecer a realidade das diferentes instituições de ensino odontológico, e tentar amenizar o problema nessa fase de aprendizado. Novos estudos com similaridade de objetivo, devem ser incentivados para expandir o conhecimento à respeito deste tema, e dessa forma, incentivar ações preventivas voltadas à saúde do trabalhador.

\section{AGRADECIMENTOS}

Financiamento: FAPESP 12/10187-8

\section{REFERÊNCIAS}

1. Biswas R, Sachdev V, Jindal V, Ralhan S. Musculoskeletal disorders and ergonomic risk factors in dental practice. Indian J Dent Sci. 2012; 4(1):70-4.

2. Coluci MZ, Alexandre NM, de Freitas Pedrini T. Musculoskeletal symptoms and workers' perception about job factors in a pulp and paper industry. Work 2012; 41(Suppl 1):5728-30.

3. Medeiros UV, Segatto GG. Lesões por esforços repetitivos (LER) e distúrbios osteomusculares (Dort) em dentistas. Rev bras odontol. 2012; 69(1):49-54.

4. Almeida GFP, Ribeiro MHA, da Silva MACN, Branco RCC, Pinheiro FGM, Nascimento MDSB. Patologias osteomusculares como causa de aposentadoria por invalidez em servidores 
públicos do município de São Luís, Maranhão. Rev Bras Med Trab. 2016; 14(1):37-44.

5. Silva EC, Camarotto JA. Contribuições da análise da atividade do trabalho no processo de retorno do trabalhador afastado por LER/DORT. Rev Ter Ocup Univ São Paulo. 2016; 27(2):131-37.

6. Azambuja MIR. Dor osteomuscular crônica problema de saúde pública que requer mais prevenção e nova abordagem clínica e previdenciária? Rev Bras Med Trab. 2012; 10(1):129-31.

7. Monteiro CR, Mancussi, Faro AC. Sintomas osteomusculares em trabalhadores de enfermagem de uma unidade neonatal, UTI neonatal e banco de leite humano. Rev Bras Med Trab. 2015; 13(2):83-90.

8. Peros K, Vodanovic M, Mestrovic S, Rosin-Grget K, Valic M. Physical fitness course in the dental curriculum and prevention of low back pain. $\mathrm{J}$ Dent Educ. 2011; 75(6):761-67.

9. Saliba TA, Machado ACB, Garbin AJI, Peruchini LFD, Garbin CAS. Análise ergonômica do atendimento clínico odontológico. Rev ABENO. 2016; 16(3): 96-105.

10. Rafie F, Zamani Jam A, Shahravan A, Raoof M, Eskandarizadeh A. Prevalence of upper extremity musculoskeletal disorders in dentists: symptoms and risk factors. J Environ Public Health. 2015; 2015:517346.

11. Ohlendorf D, Erbe C, Hauck I, Nowak J, Hermanns I, Ditchen D et al. Kinematic analysis of work-related musculoskeletal loading of trunk among dentists in Germany. BMC Musculoskelet Disord. 2016; 17(1):427

12. Thornton LJ, Barr AE, Stuart-Buttle C, Gaughan JP, Wilson ER, Jackson AD et al. Perceived musculoskeletal symptoms among dental students in the clinic work environment. Ergonomics. 2008; 51(4):573-86.

13. Presoto CD, Carrocher PA, Campos JADB, Garcia PPNS. Risk factors for musculoskeletal disorders at the workplaces of undergraduate dental students. Pesq Bras Odontoped Clin Integr 2012; 12(4):549-54.

14. Neves CP, Ribeiro DM. Burnout em estudantes de graduação em Odontologia. Rev ABENO. 2016; 16(1):39-49.

15. de Barros EN, Alexandre NM. Cross-cultural adaptation of the Nordic musculoskeletal questionnaire. Int Nurs Rev 2003; 50(2):101-8.

16. Giordano PC, Alexandre NM, Rodrigues RC, Coluci MZ. The pain disability questionnaire: a reliability and validity study. Rev Lat-Am Enfermagem. 2012; 20(1):76-83.

17. Yoon J, Choi KH, Kim TW, Yang SY, Sim MK. Reliability and validity of the korean version of the pain disability questionnaire. Ann Rehabil Med. 2013; 37(6):814-23.
18. Saliba TA, Machado AC, Marquesi C, Garbin AJ. Musculoskeletal disorders and quality of life of dentists. Rev dor. 2016; 17(4):261-65.

19. Gatchel RJ, Mayer TG, Theodore BR. The pain disability questionnaire: relationship to one-year functional and psychosocial rehabilitation outcomes. J Occup Rehabil. 2006; 16(1):75-94.

20. Anagnostis C, Gatchel RJ, Mayer TG. The pain disability questionnaire: a new psychometrically sound measure for chronic musculoskeletal disorders. Spine (Phila Pa 1976). 2004; 29(20):2290-302.

21. Carleton RN1, Abrams MP, Kachur SS, Asmundson GJ. A comparison of anatomical pain sites from a tertiary care sample: evidence of disconnect between functional and perceived disability specific to lower back pain. Eur J Pain. 2010; 14(4):410-7.

22. Geisser ME1, Robinson ME, Miller QL, Bade SM. Psychosocial factors and functional capacity evaluation among persons with chronic pain. $\mathrm{J}$ Occup Rehabil. 2003; 13(4):259-76.

23. Turner JA, Franklin G, Heagerty PJ, Wu R, Egan $\mathrm{K}$, Fulton-Kehoe D. The association between pain and disability. Pain. 2004; 112(3):307-14.

\section{CONFLITO DE INTERESSES}

Os autores declaram não haver conflitos de interesse.

\section{AUTOR PARA CORRESPONDENCIA}

\section{Renata Colturato Joaquim Gatto}

renata_colturato@hotmail.com

Submetido em 25/06/2018 Aceito em 23/08/2018 